\title{
UK ACHD response to COVID-19: Which adult patients are being advised to follow shielding by UK centres?
}

\section{Letter to the Editor}

Cite this article: Anthony JM, Kasargod Prabhakar CR, Clift P, and Hudsmith L (2020) UK ACHD response to COVID-19: Which adult patients are being advised to follow shielding by UK centres? Cardiology in the Young $\mathbf{3 0}$ : 1547-1548. doi: 10.1017/S1047951120001535

Received: 4 May 2020

Accepted: 16 May 2020

First published online: 9 June 2020

Author for correspondence: James Anthony, Clinical Nurse Specialist in Adult Congenital Heart Disease, ACHD Unit, Nuffield House, Queen Elizabeth Hospital Birmingham, Birmingham B15 2TH, UK. Tel: +44 121371 3165; Fax: +44121 371 4496. E-mail: James.anthony@uhb.nhs.uk

This important and topical letter covers the response to COVID-19 in the UK ACHD centres as to whether more patients are being advised to shield than the UK Government list on conditions, which only includes CHD if the patient is pregnant also. These data show a significant difference in approach by different UK centres.
James M. Anthony 10 , Chethan R. Kasargod Prabhakar, Paul Clift and Lucy Hudsmith

Adult Congenital Heart Disease Unit, University Hospitals Birmingham, Birmingham, UK

\section{Dear Editor,}

As COVID-19 spreads across the world, Governments and public health bodies have been advising social distancing to reduce the community transmission along with self-isolation for those who have symptoms and their close contacts. ${ }^{1}$ On 22 March, the UK Government announced that a group of "extremely vulnerable" patients would be contacted, advising them on "shielding" themselves by not leaving the home for 12 weeks. ${ }^{2}$ Shielding not only advises the patient to stay home at all times, but also to keep distance from others in the household. ${ }^{3}$

However, in the list of patients identified by the Government, patients with adult congenital heart disease (ACHD) were only identified if they were pregnant (unless they also fell into a different criteria). ${ }^{2}$ The British Congenital Cardiac Association published a statement identifying patients they considered at the greatest risk but not recommending shielding. ${ }^{4}$ We sought to establish what centres in the UK were advising their patients.

A questionnaire was sent to ACHD nurse specialists via e-mail. This asked if their centre was following the Government's recommendation on shielding, and if not, what conditions they had identified for patients that were being advised to shield.

Responses were received from all 11 level 1 UK Adult Congenital Heart Disease Surgical Centres, as well as 3 level 2 centres. 6 level 1 centres (54.5\%) advised additional patients to those who were pregnant, with all the level 2 doing the same. For those centres that had gone beyond this, there were a variety of conditions being taken into consideration. All those centres included patients with a functional single ventricle/Fontan circulation and those with chronic cyanosis such as Eisenmenger syndrome as extremely vulnerable, and a large group included pulmonary hypertension. Many also assess others on a case-by-case basis in addition.

With an absence of published data on the risk of COVID-19 on ACHD patients, centres had to make recommendations to patients quickly based on limited available guidance and their own expert and consensus opinion.

There is also as yet no evidence on the impact of shielding, not only in terms of its ability to reduce risk of infection or the potential harms from increased social isolation and decreased physical activity.

A lack of a consensus across centres may have contributed to the UK Government not including these patients in their official list of those extremely vulnerable, which has impacted on some patients' access to services such as pharmaceutical and provision deliveries. Also different advice from different centres risks confusion as patients become aware of this.

Following this study, the British Congenital Cardiac Association issued a statement which recognised that different centres had been advising shielding and advised of a formal process for adding these patients to the registry of extremely vulnerable patients. ${ }^{5}$

Further work is needed to assess the impact of COVID-19 on ACHD patients, as well as the effect of social distancing or shielding. This will be important to help the ACHD teams to advise and support patients regarding COVID-19, but also in the work of future pandemic planning.

Acknowledgements. Thanks to the British Adult Congenital Cardiac Nurses Association.

Financial support. This research received no specific grant from any funding agency, commercial or not-for-profit sectors.

Conflicts of interest. None.

\section{References}

1. Mahase E. COVID-19: WHO declares pandemic because of "alarming levels" of spread, severity, and inaction. BMJ 2020; 368: m1036.

2. UK Government. Major New Measures to Protect People at Highest Risk from Coronavirus (Press Release). Retrieved April 13, 2020, from https:/www.gov.uk/government/news/major-new-measures-to-protectpeople-at-highest-risk-from-coronavirus

3. Public Health England. Guidance on Shielding and Protecting People Defined on Medical Grounds as Extremely Vulnerable from COVID-19. Retrieved April 13, 2020, from https://www.gov.uk/government/ 
publications/guidance-on-shielding-and-protecting-extremely-vulnerablepersons-from-covid-19/guidance-on-shielding-and-protecting-extremelyvulnerable-persons-from-covid-19

4. British Congenital Cardiac Association. BCCA COVID-19 Guidance for Vulnerable Groups with Congenital Heart Disease (18 March 2020).
Retrieved April 13, 2020, from https://www.bcca-uk.org/pages/news_box. asp?NewsID=19495710

5. British Congenital Cardiac Association. BCCA Statement: Extremely Vulnerable CHD Patients Beyond the UK Governments Defined Groups (8 April 2020). 